\title{
Case report: primary resistance to osimertinib in erlotinib-pretreated lung adenocarcinoma with EGFR T790 M mutation
}

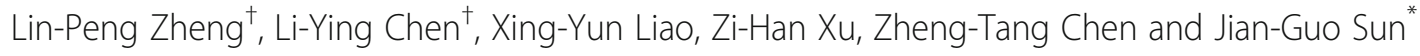

\begin{abstract}
Background: Among non-small cell lung cancer (NSCLC) patients with acquired T790 M mutation resistance to first-generation epidermal growth factor receptor-tyrosine kinase inhibitor (EGFR-TKI), 71\% are likely to benefit from osimertinib. There have been several reports about the secondary resistance to osimertinib treatment in T790 Mpositive patients, while primary resistance to osimertinib has been rarely reported.

Case presentation: A 62-year-old Asian male never smoker who presented with stage IV EGFR L858R-positive adenocarcinoma developed EGFR T790 M mutation after 14 months of treatment with erlotinib combined with thoracic radiotherapy as first-line therapy. The patient was initiated on osimertinib treatment with T790 M mutation detected (14.4\%), but disease progressed 2 months later.

Conclusion: The mechanism of primary resistance to osimertinib remains unclear. There may be an association between T790 M mutation disappearance, TP53 mutation and radiotherapy, but further researches are needed to confirm this.
\end{abstract}

\section{Backgound}

AURA3 study showed that the patients who failed in the first-generation EGFR-TKI therapy acquired 10.1 months of median PFS (mPFS) after taking osimertinib [1]. However, some of them may also resist to osimertinib after a few months, which was termed secondary resistance. To our knowledge, there have been rare reports about primary resistance to osimertinib. Herein, we report a case of primary resistance to osimertinib.

\section{Case description}

A 62-year-old male never smoker presented with several painless but slowly enlarging lymph nodes in the bilateral neck in December 2014. After a series of examinations (Fig. 1a-c), the patient was diagnosed

\footnotetext{
* Correspondence: sunjg09@aliyun.com

${ }^{\dagger}$ Lin-Peng Zheng and Li-Ying Chen contributed equally to this work. Department of Oncology, Xinqiao Hospital, Army Medical University, Chongqing 400037, China
}

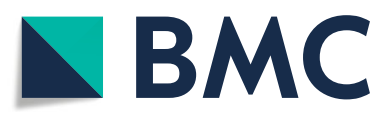

(c) The Author(s). 2018 Open Access This article is distributed under the terms of the Creative Commons Attribution 4.0 International License (http://creativecommons.org/licenses/by/4.0/), which permits unrestricted use, distribution, and reproduction in any medium, provided you give appropriate credit to the original author(s) and the source, provide a link to the Creative Commons license, and indicate if changes were made. The Creative Commons Public Domain Dedication waiver (http://creativecommons.org/publicdomain/zero/1.0/) applies to the data made available in this article, unless otherwise stated.

with lung adenocarcinoma of the left upper lobe (stage IV, cT2N3M1b) harboring L858R mutation in exon 21 of EGFR gene in January, 2015.

The patient was recruited to a clinical trial (NCT $02353741)$ and administered with erlotinib $(150 \mathrm{mg} / \mathrm{d})$ plus radiotherapy in left lung and mediastinum (PGTV60Gy/30F/6W) from January 8, 2015. Partial response (PR) was identified in this patient according to the Response Evaluation Criteria in Solid Tumors (RECIST) (version 1.1).

Disease progressed in March 2016. Neck CT found enlarged right supraclavicular nodules and axillary lymph nodes (Fig. 1d). Resection biopsy of the right supraclavicular lymph node found EGFR T790 M mutation in exon 20 (detected by ARMS-qPCR), but the lung lesions did not change much (Fig. 1d). Therefore, local radiotherapy was adopted. After following up from April 7, 2016 to January 4, 2017, the tumor response was assessed and stable disease (SD) was achieved. 

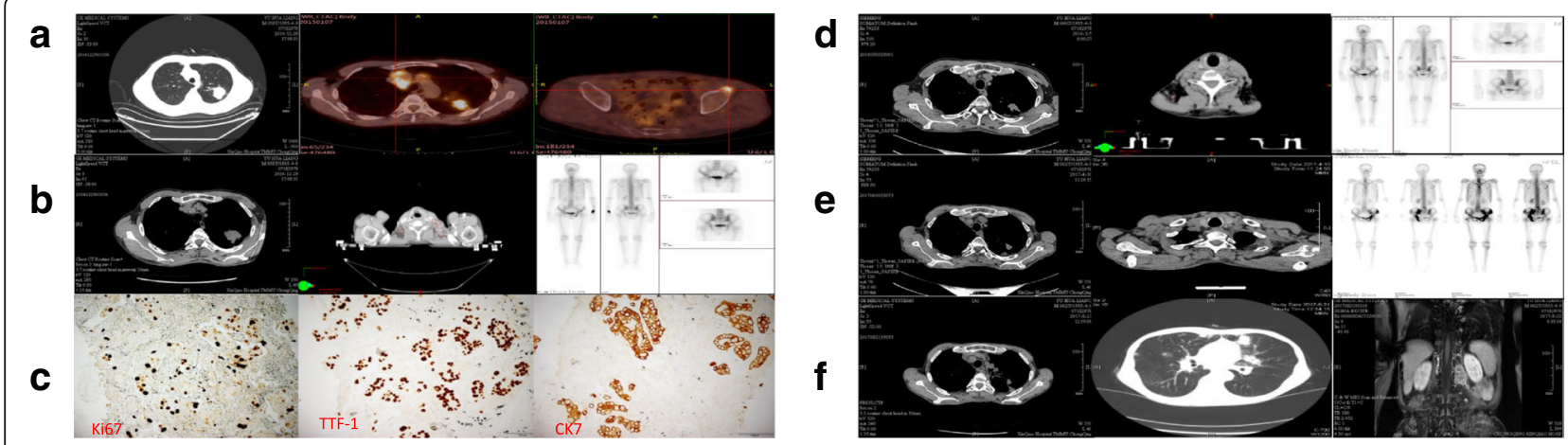

Fig. 1 diagnosis (cT2N3M1b) and tumor response in the first-, second-, third-, and fourth-line treatments. The first-line treatment (erlotinib plus radiotherapy, December 2014) (a/b) CT scans showed a mass $(3.8 \mathrm{~cm} * 3.3 \mathrm{~cm}$ ) on the left upper lobe on December 29 , 2014; PET/CT found the mass and multiple enlarged lymph nodes at bilateral neck, clavicle, left pulmonary portal, mediastinum, the 4th thoracic vertebra and left acetabulum, and showed left sciatic metastasis; Whole-body bone scintigraphy showed abnormal metabolism of the anterior superior iliac spine; (c) Immunohistochemistry staining showed high expression of CK7, TTF-1 and Ki67. Original magnification $\times 200$. $\mathbf{d}$ The second-line treatment (local radiotherapy, March 2016). Thoracic CT and Whole-body bone scintigraphy showed the lung mass and bone metastases were stable, but bilateral neck and right supraclavicular lymph nodes were slightly larger than before. e The third-line treatment (osimertinib plus local radiotherapy, April 2017). Whole-body bone scintigraphy found more bone metastases. Thoracic CT showed the lung mass was stable but cervical lymph nodes reappeared. $\mathbf{f}$ The fourth-line treatment (apatinib, August 2017). Thoracic CT showed the mass and nodules (especially lesion in the upper lobe of the left lung) were bigger. Lumbar MRI showed a mass of $5.5 \mathrm{~cm} * 2.9 \mathrm{~cm}$ in the left appendage area of the 1st and 2nd lumbar

Pelvis magnetic resonance imaging (MRI) and whole-body bone scintigraphy (Fig. 1e) showed multiple bone metastases in April 2017. Resection biopsy of supraclavicular lymph node revealed that there was no pathological transformation. Peripheral blood molecular detection found EGFR T790 M mutation (14.4\%). Thus, the patient received second-line treatment with oral osimertinib (80 mg/day) combined with radiotherapy of bilateral ischia (PGTV 54Gy/18F). No other systemic therapy was added.

However, thoracic CT identified pulmonary nodule progression (progressive disease, PD) two months later, and the patient's performance status (PS) didn't improve. Resection biopsy of the left axillary lymph node showed that EGFR L858R mutation still existed, but T790 M mutation disappeared. Erlotinib combination with pemetrexed for two cycles from July 4, 2017. A mass of $5.5 \mathrm{~cm} * 2.9 \mathrm{~cm}$ growing from the left paravertebral soft tissues of L1-2 and enlarged retroperitoneal lymph nodes in the pelvis were found on August 21, 2017 (Fig. 1f). Core needle biopsy of paravertebral mass revealed no pathological transformation of SCLC (CK +, TTF-1 +, LCA -, Ki-67 50\%+). EGFR T790 M mutation was still negative and L858R was positive. The patient was switched to apatinib, a VEGFR2 inhibitor, from August 29, 2017. However, a large amount of pleural effusion was found on September 7, 2017, and PS was 4. One month later, the patient died. A brief introduction to the treatment history was shown in Fig. 2.

\section{Discussion}

The mechanism of acquired resistance to osimertinib includes C797S mutation, T790 M disappearance, EGFR amplification, bypass pathway activation, HER2 and MET amplification, phenotypic alterations and so on [2]. Few cases of primary resistance to osimertinib forT790 M mutation have been reported, so we present one case here. In this case, T790 M mutation was positive at first, but became negative after osimertinib treatment (Fig. 3a). So T790 M mutation disappearance could be a possible mechanism of osimertinib resistance. R. Minari [3] reported that SCLC transformation might be the cause of primary resistance to osimertinib. In this case, neuron-specific enolase (NSE) level of the patient was progressively increasing, and the CEA level was slowed down after EGFR-T790 M became negative (Fig. 3b). However, pathological examination confirmed that it was still adenocarcinoma, indicating that small-cell lung cancer transformation did not occur in this case. Next generation sequencing (1021 Gene panel from Geneplus China Corp.) of the left axillary lymph node tissue after failure of osimertinib therapy found eight mutated genes (EGFR L858R44.6\%, TP53 40.0\%, ABL2 29.1\%, FAT1 27.3\%, NF2 16.2\%, CSMD3 8.9\%, RET 8.9\%, OR6F1 1.0\%). But only TP53 mutation seems 


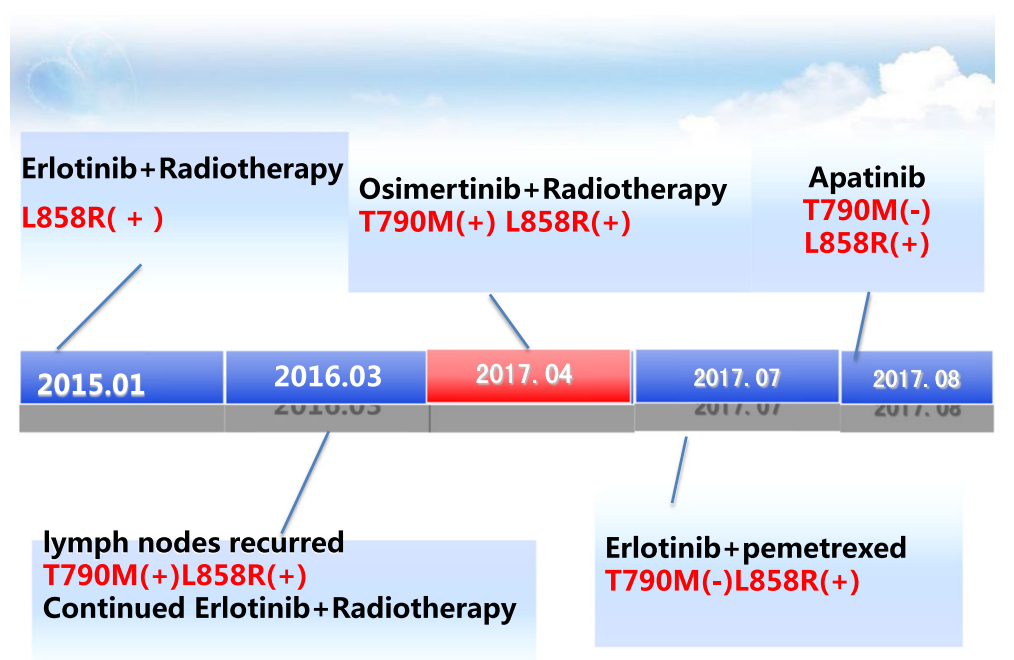

Fig. 2 Treatment history

significant. We could not find co-existing mutations like PI3K-AKT pathway. Matteo Canale [4] reported that the risk of disease progression was three times higher in patients with TP53 mutations than in those without TP53 mutation. Thus we wonder if TP53 nutation is another possible mechanism of this case.

What is more, we found the abundance of T790 M and L858R mutation dropped from 11.7 to $0.00 \%$ and from 49.8 to $34.5 \%$ after taking osimertinib, respectively, while the disease continuously progressed. Thus, the result showed that there was no relation between the decrease of EGFR mutation abundance and tumor response. Zhang, B.O [5] found ddPCR was more sensitive in detecting EGFR mutation, especially for low abundance. In this case, the patient was diagnosed with EGFR T790 M-negative mutation using ARMS-qPCR, but retrospective detection using ddPCR found that EGFR T790 M mutation was positive $(0.25 \%)$. So, if low abundance of EGFR mutation is observed, ddPCR is recommended.

Our clinical trial proves it's a good strategy to perform concurrent local radiotherapy for first-line EGFR-TKI and local progression in advanced NSCLC patients [6]. In this case, the patient received concurrent erlotinib with local radiotherapy as first-line therapeutic strategy and the PFS was 14 months. However, primary resistance to osimertinib was observed in this patient. Thus, we wonder if radiotherapy plays a role in primary resistance to osimertinib. Hirata $\mathrm{H}$ and his colleagues [7] reported that acquired resistance to TKIs appears to be associated with low efficacy of radiotherapy, but there are no reports about the relationship between primary resistance
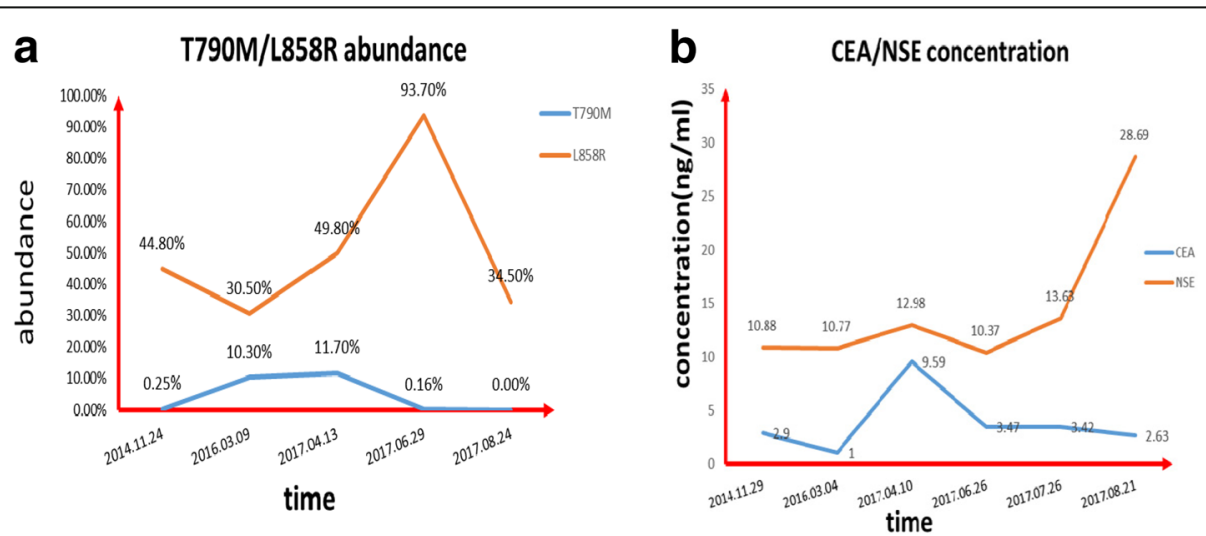

Fig. 3 Molecular and pathological analysis. a Droplet digital polymerase chain reaction (ddPCR) for retrospective detection of the EGFR L858R and T790 M mutation abundance (tumor tissue). T790 M mutation abundance was $0.25 \%$ when the patient was diagnosed, $11.7 \%$ when he began to take osimertinib, $0.16 \%$ two months after taking osimertinib, and $0 \%$ four months after taking osimertinib. b Neuron-specific enolase (NSE) level was progressively increasing. CEA level reached peak in June 2017 
to osimertinib and radiotherapy. In conclusion, this is a case report of primary resistance to osimertinib in erlotinib-pretreated lung adenocarcinoma with EGFR T790 M mutation. The mechanism is unclear. T790 M mutation disappearance, TP53 mutation and radiotherapy could be associated.

\section{Abbreviations}

ddPCR: Droplet digital polymerase chain reaction; EGFR-TKI: Epidermal growth factor receptor tyrosine kinase inhibitor; NGS: Next generation sequencing; NSCLC: Non-small cell lung cancer; NSE: Neuron specific enolase

\section{Acknowledgements}

The authors thank all our colleagues who helped us with outcome data collection.

\section{Funding}

This study was supported by the National Natural Science Foundation of China (No. 81672841), Clinical Innovation Foundation of Army Medical University (yclkt-201408) and Wu Jieping Medical Foundation (320.6799.15037).

\section{Availability of data and materials}

All relevant data are within the paper.

\section{Authors' contributions}

LPZ and LYC contributed equally. LYC, ZTC, JGS were involved in the clinical management of the patient. ZHX and XYL contributed imaging data collection. Lin-Peng Zheng wrote the main structure of the manuscript. JGS, $X Y L$ revised the manuscript. All authors read and approved the final manuscript.

\section{Ethics approval and consent to participate}

The study was approved by the ethics committee of the Xinqiao Hospital of Army Medical University. The tissue samples used in this study have been collected in Xinqiao Hospital of Army Medical University. We obtained written informed consent from the patient prior to the study.

\section{Consent for publication}

Written informed consent was obtained from the patient's daughter for publication of this case report.

\section{Competing interests}

The authors declare that they have no competing interests.

\section{Publisher's Note}

Springer Nature remains neutral with regard to jurisdictional claims in published maps and institutional affiliations.

Received: 28 February 2018 Accepted: 24 October 2018

Published online: 06 November 2018

\section{References}

1. Mok TS, Wu Y, Ahn M, et al. Osimertinib or platinum-Pemetrexed in EGFR T790M-positive lung Cancer. N Engl J Med. 2017;376:629-40.

2. Minari R, Bordi P, Tiseo M. Third-generation epidermal growth factor receptor-tyrosine kinase inhibitors in T790M-positive non-small cell lung cancer: review on emerged mechanisms of resistance. Transl Lung Cancer Res. 2016;5:695-708.

3. Minari R, Bordi P, Del RM, et al. Primary resistance to osimertinib due to SCLC transformation: issue of T790M determination on liquid re-biopsy. Lung Cancer. 2018;115:21-7.

4. Canale M, Petracci E, Delmonte A, et al. Impact of TP53 mutations on outcome in EGFR-mutated patients treated with first-line tyrosine kinase inhibitors. Clin Cancer Res. 2017;23:2195-202.

5. Zhang BO, Xu CW, Shao Y, et al. Comparison of droplet digital PCR and conventional quantitative PCR for measuring EGFR gene mutation. Exp Ther Med. 2015;9:1383-8.
6. Wang $Y, L i Y, X i a L$, et al. Continued EGFR-TKI with concurrent radiotherapy to improve time to progression (TTP) in patients with locally progressive non-small cell lung cancer (NSCLC) after front-line EGFR-TKI treatment. Clin Transl Oncol. 2017;20(3):366-73.

7. Hirata H, Nakamura K, Kunitake N, et al. Association between EGFRTKI resistance and efficacy of radiotherapy for brain metastases from EGFR-mutant lung adenocarcinoma. Anticancer Res. 2013;33:1649-55.

\section{Ready to submit your research? Choose BMC and benefit from:}

- fast, convenient online submission

- thorough peer review by experienced researchers in your field

- rapid publication on acceptance

- support for research data, including large and complex data types

- gold Open Access which fosters wider collaboration and increased citations

- maximum visibility for your research: over $100 \mathrm{M}$ website views per year

At BMC, research is always in progress.

Learn more biomedcentral.com/submissions 